\title{
Penetration of Topical Glucosamine Sulfate into the Synovial Fluid of Patients with Knee Osteoarthritis: A Nonrandomized, Open-Label, Single Dose, Bioavailability Study
}

\author{
Matthew Kong1, Khadijah Binte Hashim"1, Phoebe Lin', Gaëlle Coestesquis ${ }^{2}$, Aiyun Xu' \\ Frederick Lebes ${ }^{2}$, Choon Meng Ting ${ }^{3}$ \\ ${ }^{1}$ School of Applied Science, Temasek Polytechnic, Singapore \\ ${ }^{2}$ Lynk Biotechnologies Pte. Ltd., Singapore \\ ${ }^{3} \mathrm{~T} \& \mathrm{~T}$ Family Health Clinic \& Surgery, Singapore \\ Email: kongcr@tp.edu.sg, rd@lynkbiotech.com
}

How to cite this paper: Kong, M., Hashim, K.B., Lin, P., Coestesquis, G., Xu, A.Y., Lebes, F. and Ting, C.M. (2019) Penetration of Topical Glucosamine Sulfate into the Synovial Fluid of Patients with Knee Osteoarthritis: A Nonrandomized, OpenLabel, Single Dose, Bioavailability Study. Journal of Biosciences and Medicines, 7, 76-90.

https://doi.org/10.4236/jbm.2019.711007

Received: October 4, 2019

Accepted: November 5, 2019

Published: November 8, 2019

Copyright $\odot 2019$ by author(s) and Scientific Research Publishing Inc. This work is licensed under the Creative Commons Attribution International License (CC BY 4.0).

http://creativecommons.org/licenses/by/4.0/

\section{(c) (i) Open Access}

\begin{abstract}
Objective: This study aims to show that a proprietary topical cream can deliver glucosamine through the skin into the synovial fluid of osteoarthritic patients. This cream contains $10 \% \mathrm{w} / \mathrm{w}$ glucosamine sulfate. It also aims to determine the endogenous level of glucosamine in the synovial fluid of these patients. Therapeutic effectiveness of glucosamine is not addressed in this study. Design: This phase IV, open-label, nonrandomized study enrolled 240 patients. Participants from the Test group received a single dose treatment ( $2 \mathrm{~g}$ of cream), and synovial fluid samples were collected 1 - 3 hours post-treatment. Patients from the Control group were not subjected to any treatment but their synovial fluid was also sampled to establish a glucosamine concentration baseline for Time-0 (T0). Glucosamine concentrations were determined by HPLC analysis. Results: The mean glucosamine concentration in the synovial fluid of patients from the Test group (100.56 $\mathrm{ng} / \mathrm{ml}, 95 \%$ CI $66.36-134.76, \mathrm{n}=117)$ was higher than in the Control group $(17.83 \mathrm{ng} / \mathrm{ml}, 95 \%$ CI $7.42-28.24, \mathrm{n}=117)$ resulting in a significant between-group difference $(\mathrm{p}<0.0001)$. While the gender of the subjects did not appear to affect the results, a significant difference was observed with age variation. Conclusion: The results suggest that glucosamine can be topically delivered across the human skin into the synovial fluid using a proper vehicle. This suggests that other water-soluble molecules could similarly be delivered transdermally, alleviating the need for oral delivery in cases where oral administration is difficult, or when harmful side effects could ensue.
\end{abstract}




\section{Keywords}

Osteoarthritis of the Knee, Transdermal Delivery, Glucosamine, Synovial Fluid, Joints

\section{Introduction}

Osteoarthritis (OA) is a highly prevalent joint disease characterized by joint cartilage degeneration, a condition that affects $9.6 \%$ of men and $18 \%$ of women over 60 years old worldwide [1].

Our joints are cushioned by cartilages and lubricated by synovial fluid such that we can move any joint freely without pain. The principal lubricating substances in our cartilage and synovial fluid are proteoglycans and glycosaminoglycans (GAGs). Glucosamine, the main ingredient needed to produce GAGs, is naturally produced by our body, but the body loses its capacity to produce sufficient glucosamine with age and causes thinning of the cartilage, leading to joint degeneration [2].

Contradictory research studies on glucosamine were published [2], and OA treatment guidelines developed by scientific organizations are often debated. Organizations such as the American College of Rheumatology (ACR) or the Osteoarthritis Research Society International (OARSI) recommend against the use of glucosamine for the treatment of OA. According to the OARSI Guidelines, glucosamine is not an efficacious disease-modifying drug for OA, and also does not guarantee outcomes as a pain reliever. However, it is important to note that transdermal delivery or skin application of glucosamine was not considered in the construct of the OARSI Guidelines. However, other organizations such as the European League Against Rheumatism (EULAR) and the European Society or Clinical and Economic Aspects of Osteoporosis and Osteoarthritis (ESCEO) recommend symptomatic slow-acting drugs such as glucosamine sulfate for the background therapy of knee OA. This was based on high-quality evidence showing that glucosamine was superior to placebos in the treatment of pain and functional impairment [3].

For targeted drug administration, it is widely established that topical administration offers numerous advantages over oral delivery [3]. The benefits of topical administration include maximizing the bioavailability of the drug, optimizing therapeutic efficacy, and minimizing side effects [4]. Topical delivery avoids the occurrence of the hepatic first-pass effect, and also has advantages over both intravenous and intramuscular routes as it is a painless and noninvasive method of drug delivery [3].

In the work of Hammad et al., the clinical efficacy of topical vs oral glucosamine/chondroitin sulfate in 180 patients with OA of the knee, were randomly assigned and compared. They found that both treatments were safe and equally effective for improving knee pain, but indicated that topical glucosamine ad- 
ministration was superior to the oral route in improving stiffness and function of the joint [2]. In a different study by Cohen et al., 63 patients suffering from $\mathrm{OA}$ of the knee were randomly divided into 2 groups. The group receiving topical glucosamine and chondroitin showed significant pain relief compared to the placebo group [5]. These papers have demonstrated the statistical relationship between the application of topical glucosamine and positive OA outcomes, but have not demonstrated that the applied glucosamine has indeed crossed the skin to reach the diseased joint.

Hence, this study is designed to produce the evidence that glucosamine did actually reach the diseased area after topical application. The aim of the present nonrandomized, open-label, single dose study was to investigate the feasibility of penetration of a $10 \%$ glucosamine cream into the synovial fluid of patients with joint effusions and intended arthrocentesis (synovial fluid aspiration). A second outcome was to determine the endogenous level (or baseline) of glucosamine in synovial fluid of osteoarthritic knees for the group with no treatment administered. The hypothesis of the study was that higher concentrations of glucosamine would be found in the synovial fluid of patients using the cream.

Whether the topical glucosamine dosage was sufficient to be translated into clinical effects was not addressed in this study and could be an objective for future work. To our knowledge, this is the first study describing the bioavailability of glucosamine in human synovial fluid following the application of a topical cream containing glucosamine.

\section{Methods}

\subsection{Ethical Considerations}

The study protocol and related materials were approved by the Institutional Review Board of Temasek Polytechnic, Singapore. The study was conducted in conformance with the current revision of the Declaration of Helsinki, and with current Good Clinical Practice, Singapore Guidelines. Written informed consent was obtained from all participants before commencement of the study. The study was registered at clinicaltrials.gov (NCT03743896) and with the Singapore Health Sciences Authority (CTC1600169). This report adheres to the Transparent Reporting of Evaluations with Non-randomised Designs (TREND) statement, which complement the widely adopted CONsolidated Standards Of Reporting Trials (CONSORT) statement [6].

\subsection{Patient Eligibility and Study Design}

A Phase IV, open-label, nonrandomized trial was designed involving volunteers with OA of the knee. To ensure that the collection of samples for the determination of glucosamine concentration would not involve additional interventions, unnecessary pain and consequent risk, patients with joint effusions and planned arthrocentesis were selected. Participants recruited were men and nonpregnant women from 21 to 80 years of age, who had been diagnosed with knee OA (stage 
2 and 3) prior to recruitment. Patients with any form of personal glucosamine supplement or pain relief treatment taken during the last 24 hours were excluded from this study. Additional exclusion criteria included known allergy to shellfish, glucosamine, and capsaicin, as well as a history of skin sensitivity, skin abnormality at application site and women who were nursing. Participants were grouped into Control (untreated) and Test (treated) groups.

The outpatient clinic, located in Singapore, specialises in arthrocentesis and viscosupplementation. The medical doctor in charge of the clinic has been performing these procedures since 1991 [7]. He is also the author of a handbook about viscosupplementation for OA knees, and in this book, he describes the ways patients are diagnosed with knee OA as well as the procedures for synovial fluid extraction and viscosupplementation [8].

\subsection{Study Intervention and Allocation}

TGC $^{\circ}$ Plus Capsaicin, a transdermal glucosamine cream containing $10 \% \mathrm{w} / \mathrm{w}$ glucosamine sulfate, is commercially available for joint pain relief, and manufactured by Lynk Biotechnologies Pte. Ltd., Singapore. It is listed as a medicinal product exempted from registration by the Singapore Health Sciences Authority. All patients taking part in the study were offered a free $45 \mathrm{~g}$ tube of TGC ${ }^{\circ}$ Plus Capsaicin. The joint fluid aspiration cost was waived for patients of the Test group.

Participants were given full discretion to decide which of the two groups they wished to be assigned to. Test group volunteers were given treatment by the clinic's nurses of a topical application of the cream ( $2 \mathrm{~g}$ single dose treatment) on the affected knee 1 - 3 hours before the extraction of the synovial fluid by the medical practitioner. The single dose of $2 \mathrm{~g}$ (200 $\mathrm{mg}$ glucosamine) was decided due to surface area limitations. A larger amount would have been impractical for topical application. The synovial fluid was collected after 1 to 3 hours, based on results from previous internal studies. In these studies, the maximum glucosamine concentration in mice plasma was obtained within this time range. The Control group volunteers were not given any treatment and proceeded directly for the extraction of fluid. This fluid, which is normally discarded by the clinic, was collected for both groups.

Since the primary endpoint is to determine the concentration of glucosamine present in the synovial fluid, there was no possibility of subjective bias or placebo effect. Hence no randomisation or patient's blinding was necessary. However, blind analyses were conducted independently by third party testing lab (Temasek Polytechnic, Singapore). Synovial fluid samples were only labelled with each participant's identification code.

\subsection{Materials}

(D)-(+)-Glucosamine hydrochloride ( $\geq 99.9 \%)$, 1-naphthyl isothiocyanate (95\%), methylene chloride (99\%), and triethylamine (99\%) were obtained from SigmaAldrich. Acetonitrile (HPLC grade, 99.9\%), methanol (HPLC grade, 99.9\%) and 
glacial acetic acid (ACS grade, 99.0\%) were obtained from Merck Millipore.

\subsection{Sample Preparation and Analyses}

The synovial fluid samples were centrifuged at $4^{\circ} \mathrm{C}, 1400 \times \mathrm{g}$ for 15 minutes immediately after collection. The cell-free supernatant was harvested and stored at $-80^{\circ} \mathrm{C}$ pending assay. The concentration of glucosamine in each synovial fluid sample was determined by a validated high-performance liquid chromatography-ultraviolet visible spectrometry (HPLC-UV) method. After thawing at room temperature, synovial fluid sample $(200 \mu \mathrm{l})$ was pipetted into a $1.5-\mathrm{ml}$ centrifuge tube and acetonitrile $(400 \mu \mathrm{l})$ was subsequently added for protein precipitation. Methanol $(200 \mu \mathrm{l})$ was then added and the resultant mixture was vortexed and centrifuged at $14,000 \times \mathrm{g}$ for 2 minutes. The supernatant was recovered and dried using a freeze dryer (Scanvac, Labogene). To the dried residue, $0.1 \mathrm{ml}$ of derivatizing reagent, 1-naphthyl isothiocyanate in methanol:acetonitrile:triethylamine (1:1:0.2) mixture at $50 \mathrm{mg} / \mathrm{ml}$ was added. The mixture was vortexed and left at ambient conditions for 30 minutes. The reaction was quenched by adding $0.2 \mathrm{ml}$ of acetic acid solution $(1.5 \% \mathrm{v} / \mathrm{v})$. The excess derivatizing reagent and its degradation products were partitioned into an organic phase by the addition of $1.0 \mathrm{ml}$ of methylene chloride. The upper aqueous layer was transferred to another 2-ml centrifuge tube and the extraction process was repeated three times. $150-170 \mu \mathrm{l}$ of the upper aqueous layer was filtered using a $0.45 \mu \mathrm{m}$ syringe filter (Thermo Scientific) and transferred into vials for HPLC analyses.

The instrument and the optimised parameters used are as follows: Agilent 1290 Liquid Chromatography system with autosampler and UV detector; Column type: Two Inertsil ODS-3 $(4.0 \mathrm{~mm} \times 250 \mathrm{~mm}, 3 \mu \mathrm{m})$ connected in sequence; Column temperature: $27^{\circ} \mathrm{C}$; Flow rate: $0.3 \mathrm{ml} / \mathrm{min}$; Injection volume: 10 $\mu \mathrm{l}$; Detection wavelength: $224 \mathrm{~nm}$; HPLC elution: Binary gradient elution with mobile phase A (80:20 ultrapure water:acetonitrile with $0.04 \% \mathrm{v} / \mathrm{v}$ acetic acid and $0.04 \% \mathrm{v} / \mathrm{v}$ triethylamine) and mobile phase B (Acetonitrile); 0 - $65 \mathrm{~min}$ (100\% A), 65 - $85 \min (100 \%$ B), 85 - $100 \min (100 \%$ A).

Due to potential matrix effects in analysing synovial fluid, the standard addition method was used to quantify the concentration of glucosamine. The standard addition method was carried out by spiking each synovial fluid sample separately with two different concentrations of standard solutions of glucosamine hydrochloride to achieve additional concentrations of glucosamine of 250 $\mathrm{ng} / \mathrm{ml}$ and $500 \mathrm{ng} / \mathrm{ml}$, respectively.

\subsection{Sample Size Estimation}

This study was not restricted by the minimum sample size requirement as the design deliberately involved a large sample size $(\mathrm{N}=240)$ to improve the confidence level of results obtained. The Control group acts as a proxy for a Time- 0 (T0) sample to establish a baseline of glucosamine level in the synovial fluid of subjects in the Test group. The clinic being specialised in synovial fluid aspira- 
tion, potential trial participants were readily available and synovial fluid samples are usually discarded. Therefore, there were no ethical concerns by having a high number of participants.

\subsection{Statistical Analysis and Outliers}

Analyses were conducted using GraphPad Software ๑ 2018. Outcomes were assessed using a univariate, unpaired Student t-test. Two-tailed P-values $<0.01$ were considered statistically significant. The Fisher's test was used for comparison of baseline categorical data.

The Z-score method was used to identify outliers in both the Control group and the Test group dataset. Samples with a Z-value greater than 3 were taken to be outliers and excluded from the concentration calculation.

\section{Results}

\subsection{Participants Disposition and Baseline Characteristics}

From May 2016 to March 2017, 240 patients undergoing arthrocentesis were selected for the study, with 120 participants in each group. The participants consisted of $58(24.17 \%)$ men and $182(75.83 \%)$ women, with mean age of $64.24 \pm$ 8.58 years old (mean $\pm \mathrm{SD}$ ). There were no notable differences in participant demographic between Control and Test groups (Table 1).

The patient enrolment process and sample analysis process are summarized in the enrolment chart (Figure 1). There was no follow up necessary and no deviation from the protocol.

Table 1. Demographic details of study participants—sex, mean age, and age group.

\begin{tabular}{|c|c|c|c|c|}
\hline Characteristics & $\begin{array}{l}\text { All participants } \\
\qquad(\mathrm{N}=240)\end{array}$ & $\begin{array}{l}\text { Test group } \\
(\mathrm{N}=120)\end{array}$ & $\begin{array}{l}\text { Control group } \\
(\mathrm{N}=120)\end{array}$ & P-value \\
\hline \multicolumn{5}{|l|}{ Sex, N (\%) } \\
\hline Female & $182(75.83)$ & $87(72.50)$ & $95(79.17)$ & \\
\hline Male & $58(24.17)$ & $33(27.50)$ & $25(20.83)$ & 0.2912 \\
\hline Mean age (SD, range), years & $\begin{array}{c}64.24 \\
(8.58,39-80)\end{array}$ & $\begin{array}{c}63.61 \\
(8.37,39-80)\end{array}$ & $\begin{array}{c}64.88 \\
(8.78,39-80)\end{array}$ & 0.2526 \\
\hline Mean age female (SD), years & $64.54(8.87)$ & $64.13(8.54)$ & $64.92(9.19)$ & 0.5498 \\
\hline Mean age male (SD), years & $63.29(7.62)$ & $62.24(7.88)$ & $64.68(7.17)$ & 0.2301 \\
\hline \multicolumn{5}{|l|}{$\begin{array}{l}\text { Age group participants, } \\
\qquad \mathrm{N} \text { female }+ \text { male }\end{array}$} \\
\hline 39 to 50 years old & $14+6$ & $5+5$ & $9+1$ & 0.1409 \\
\hline 51 to 60 years old & $47+10$ & $28+3$ & $19+7$ & 0.1602 \\
\hline 61 to 70 years old & $72+33$ & $35+23$ & $37+10$ & 0.0574 \\
\hline 71 to 80 years old & $49+9$ & $19+2$ & $30+7$ & 0.4647 \\
\hline
\end{tabular}

Abbreviations: $\mathrm{SD}=$ Standard Deviation. 


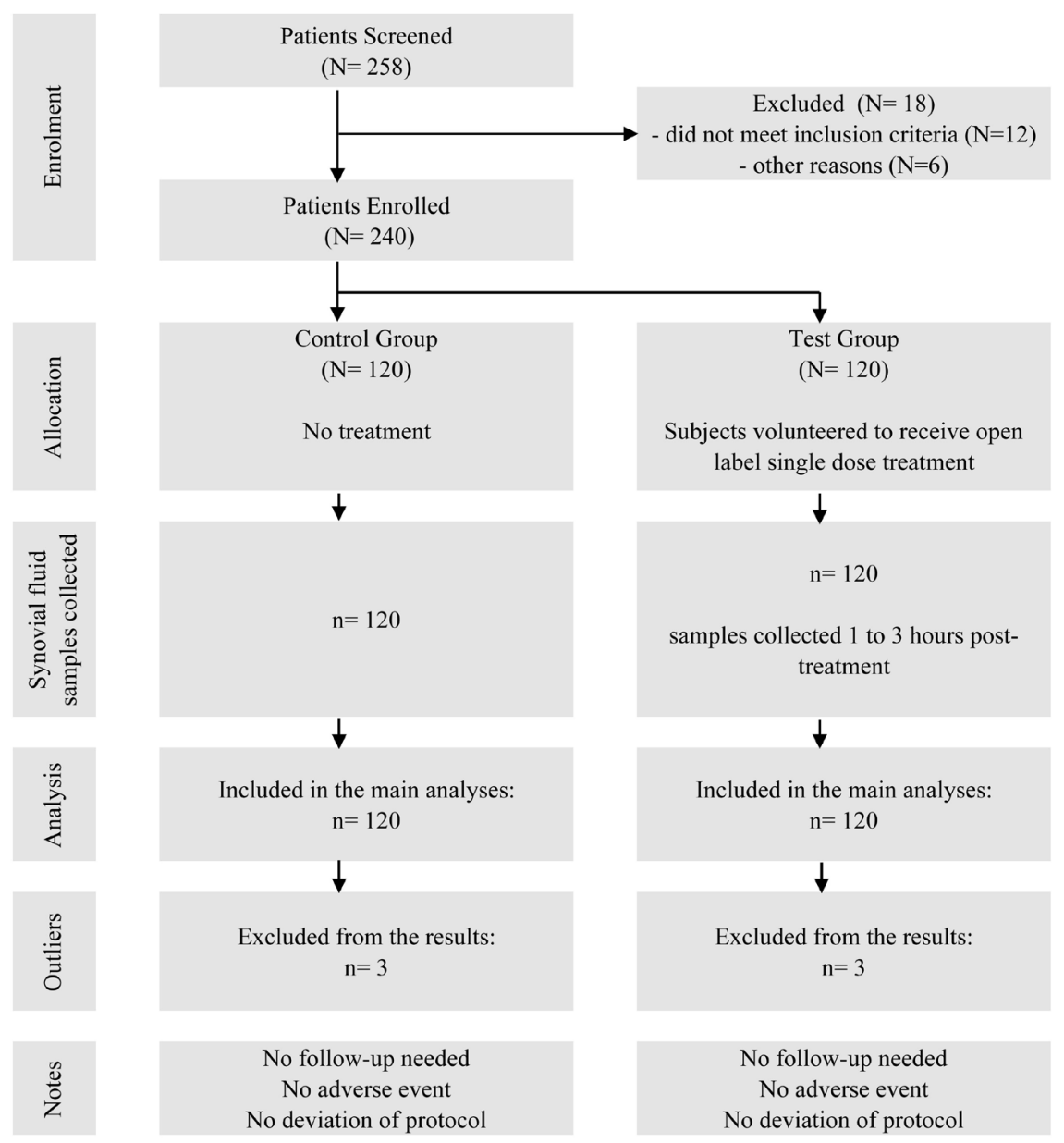

Figure 1. Enrolment chart. 120 participants were selected for each group. All synovial fluid samples were included in the main analysis.

A total of 240 samples of synovial fluid were collected. The Control group, consisting of 120 samples, were not subjected to any treatment prior to arthrocentesis. The Test group, with a total of 120 samples, were treated with a single dose $(2 \mathrm{~g})$ of topical glucosamine cream 1 to 3 hours prior to arthrocentesis.

\subsection{Overall Results-Test Group vs Control Group}

In Table 2, the HPLC results show that the mean concentration of glucosamine of the Test group was $100.56 \mathrm{ng} / \mathrm{ml}(95 \%$ CI $66.36-134.76, \mathrm{n}=117)$ and is statistically higher $(\mathrm{P}<0.0001)$ than that of the corresponding value for the Control group $(17.83 \mathrm{ng} / \mathrm{ml}, 95 \% \mathrm{CI} 7.42-28.24 ; \mathrm{n}=117)$. Between 1 and 3 hours post-treatment, the mean concentration of glucosamine measured from the Test group was more than five times than that of the Control group, demonstrating a significant difference between samples from the two groups.

Figure 2 is a graphical representation of glucosamine concentrations in the synovial fluid of all subjects from both groups. It is evident that more subjects in the Test group have significantly higher levels of glucosamine in synovial fluid compared to the Control group. Of the Control group, 96 of the 117 samples 


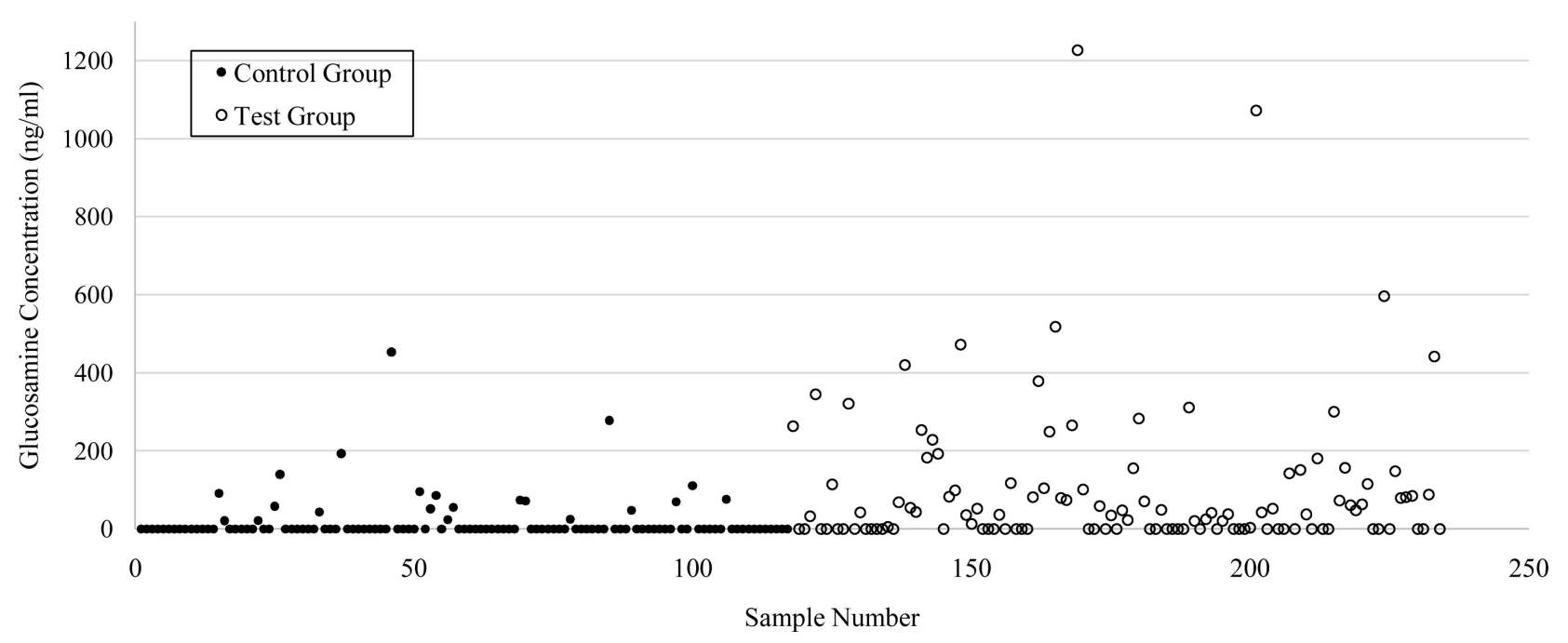

Figure 2. Distribution of glucosamine concentrations (in $\mathrm{ng} / \mathrm{ml}$ ) in synovial fluid samples of subjects from the Control group and the Test group. The comparison shows higher concentrations of glucosamine present in samples of Test group subjects compared to samples of Control group subjects.

Table 2. Mean glucosamine concentrations of test and control groups.

\begin{tabular}{ccc}
\hline \multirow{2}{*}{ Overall Results } & Glucosamine concentration $(\mathrm{ng} / \mathrm{ml})$ & \multirow{2}{*}{ P-value } \\
\cline { 2 - 3 } & Mean $(95 \% \mathrm{CI})$ & \\
\hline Control $(\mathrm{n}=117)$ & $17.83(7.42-28.24)$ & $<0.0001$ \\
Test $(\mathrm{n}=117)$ & $100.56(66.36-134.76)$ & \\
\hline
\end{tabular}

Abbreviation: $\mathrm{CI}=$ Confidence Interval.

(82\%) have no detectable glucosamine, while only 48 samples in the Test group (41\%) have no detectable glucosamine. In addition, 18 samples of the Test group (15.3\%) had a measured glucosamine concentration of $>200 \mathrm{ng} / \mathrm{ml}$, compared to only 2 samples of the Control group (1.7\%) surpassing this concentration. This difference in detectable levels of glucosamine between the two groups implies that a sufficient amount of glucosamine was absorbed through the skin and delivered to the subject's synovial fluid within one to three hours after the application of topical glucosamine.

\subsection{Results-By Sex}

Table 3(a) shows the glucosamine concentrations in synovial fluid samples from subjects in both groups, categorised by sex. There was a significant difference between Test and Control groups for both male and female subjects. However, the difference between male $(136.94 \mathrm{ng} / \mathrm{ml}, 95 \%$ CI $61.48-212.40 ; \mathrm{n}=33)$ and female $(86.26 \mathrm{ng} / \mathrm{ml}, 95 \%$ CI 48.33 - 124.20; $\mathrm{n}=84)$ Test Groups was not statistically significant $(\mathrm{P}=0.1878)$.

\subsection{Results-By Age Group}

Table 3(b) shows the glucosamine concentrations in synovial fluid samples from subjects classified by age groups of 39 to 60 years old and 61 to 80 years old. The 
data indicates a significant difference between Test and Control groups for the older age group $(\mathrm{P}<0.0001)$, but not for the younger age group $(\mathrm{P}=0.0896)$.

When subjects were further categorised into specific age groups with a narrower 10-year interval, glucosamine concentrations for Test and Control subjects in the age groups of $39-50$ years old $(\mathrm{P}=0.8044)$ and $51-60$ years old $(\mathrm{P}=$ $0.0879)$ were not statistically significant. However, significant differences were observed among subjects in the advanced age group of $61-70$ years old $(\mathrm{P}=$ $0.0014)$ and $71-80$ years old $(\mathrm{P}=0.0019)$ showing more pronounced differences in glucosamine concentration. While the glucosamine concentration remains relatively constant for all the Control group participants, a different trend of data among participants of the Test group suggests that glucosamine concentration in the synovial fluid, following application of topical glucosamine, increases with age (Figure 3).

Table 3. Mean glucosamine concentrations of test and control groups: (a) by sex, (b) by age group.

\begin{tabular}{|c|c|c|c|c|}
\hline \multirow[t]{2}{*}{ Category } & & \multirow[t]{2}{*}{ Treatment Group } & $\begin{array}{c}\text { Glucosamine } \\
\text { Concentration }(\mathrm{ng} / \mathrm{ml})\end{array}$ & \multirow[t]{2}{*}{ P-value } \\
\hline & & & Mean (95\% CI) & \\
\hline \multirow{2}{*}{ (a) By sex } & Female & $\begin{array}{c}\text { Control }(n=93) \\
\text { Test }(n=84)\end{array}$ & $\begin{array}{c}17.34(5.13-29.55) \\
86.26(48.33-124.20)\end{array}$ & 0.0004 \\
\hline & Male & $\begin{array}{l}\text { Control }(n=24) \\
\text { Test }(n=33)\end{array}$ & $\begin{array}{c}19.73(-0.17-39.63) \\
136.94(61.48-212.41)\end{array}$ & 0.0106 \\
\hline \multirow{2}{*}{ (b) By age group } & $\begin{array}{l}39 \text { to } 60 \\
\text { years old }\end{array}$ & $\begin{array}{l}\text { Control }(n=33) \\
\text { Test }(n=40)\end{array}$ & $\begin{array}{c}14.82(3.60-26.03) \\
46.96(13.94-79.97)\end{array}$ & 0.0896 \\
\hline & $\begin{array}{l}61 \text { to } 80 \\
\text { years old }\end{array}$ & $\begin{array}{l}\text { Control }(\mathrm{n}=84) \\
\text { Test }(\mathrm{n}=77)\end{array}$ & $\begin{array}{c}19.01(5.08-32.95) \\
128.40(80.00-176.81)\end{array}$ & $<0.0001$ \\
\hline
\end{tabular}

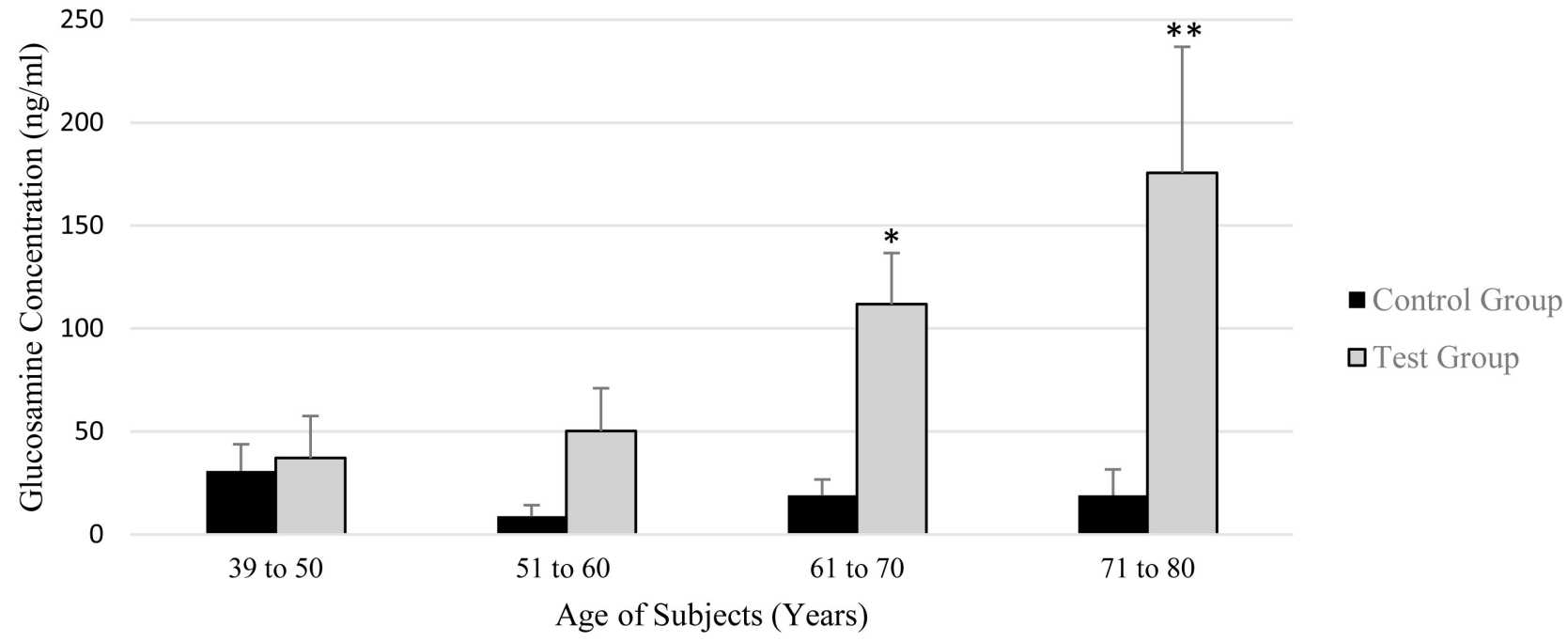

Figure 3. Distribution of glucosamine concentrations in synovial fluid samples of Control and Test group subjects by 10 -year age groups $\left({ }^{\star} P=0.0014 ;{ }^{\star *} P=0.0019\right)$. The results show that glucosamine levels increase significantly with age. 


\subsection{Safety}

There were no allergic or adverse reactions reported during or after the application of the glucosamine cream by participants of the Test group.

\section{Discussion}

\subsection{Overview}

The results of the study present empirical evidence of glucosamine bioavailability and transdermal delivery into human synovial fluid after application of a topical glucosamine cream on knees of osteoarthritic patients.

Observations show that the mean glucosamine concentration in the synovial fluid of subjects from the Test group was significantly higher than the glucosamine concentration in the synovial fluid of subjects from the Control group. These results are corroborated by another study assessing the glucosamine concentration in synovial fluid after 14-day treatment of $1500 \mathrm{mg}$ oral glucosamine [9]. This study reported a median glucosamine concentration of $777 \mathrm{ng} / \mathrm{ml}$ for synovial fluid samples collected 3 hours after the last dose of the 2-week treatment. Our study shows that with a single dose of cream (containing $200 \mathrm{mg}$ glucosamine) applied on skin, a mean glucosamine concentration of 100.56 $\mathrm{ng} / \mathrm{ml}$ can be detected. Correlations of absorbed glucosamine levels in synovial fluid to demographic information of the patients, such as age and sex, were also studied.

\subsection{Endogenous Glucosamine Level}

When subjects in the Control group were categorised by sex or age, it was observed that the mean glucosamine concentrations were within a consistent range. It can be seen that the baseline (endogenous) glucosamine concentration in synovial fluid does not vary across sex and age. In this study, the glucosamine concentration from subjects in the Control group $(17.83 \mathrm{ng} / \mathrm{ml}, 95 \%$ CI 7.42 28.24; $n=117$ ) regardless of sex or age is similar to the results from two previous studies, where the endogenous glucosamine concentrations in synovial fluid were reported to be $36.5 \mathrm{ng} / \mathrm{ml}$ [9] and $32.3 \mathrm{ng} / \mathrm{ml}$ [10].

\subsection{Impact of Sex on Glucosamine Absorption}

All participants in the Test group were treated with a uniform dose $(2 \mathrm{~g})$ of transdermal glucosamine cream. The trend of absorbed glucosamine in relation to sex was investigated and it was observed that there was a significant difference between Test and Control groups for both sexes. This suggested that in the Test group, both male and female participants (of all ages) showed similar positive responses after the application of the cream. Although data showed the mean glucosamine concentrations in male participants appeared to be higher than in female participants in the Test group, it was not statistically significant and did not demonstrate an apparent difference between genders. Other studies involving transdermal absorption of compounds from topical application also showed 
that the degree or rate of absorption were not dependant on gender [11] [12]. Similar to these studies, this transdermal study of topical glucosamine application also showed that it was not selective and independent of sex.

\subsection{Impact of Age on Glucosamine Absorption}

A possible correlation with participants' age was also considered in this study. The approach employed was grouping the subjects into two age categories of 39 - 60 years old and 61 - 80 years old, and analysing the glucosamine concentration of each subject within each age category. The glucosamine level of younger Test group patients did not significantly increase following treatment $(\mathrm{P}=$ 0.0896). However, the older Test group patients were found to have a significantly higher level of glucosamine $(\mathrm{P}<0.0001)$ compared to the control group. As shown in Figure 3, an increasing trend in glucosamine levels, as a function of age, became more prominent when age groupings were modified into more specific age categories of 10-year intervals. The results suggested that the rate of transdermal glucosamine transport increases with age and that transdermal delivery of glucosamine might have higher rates in people of advanced age than their younger counterparts. More data would be needed to confirm this trend as the population of younger patients was limited in this study.

A direct correlation of transdermal delivery rate with skin ageing was postulated in this work. It has been reported that skin thins progressively at an accelerating rate with age [13], and the thinning of skin tissue may allow better transdermal absorption of glucosamine. Konda and colleagues reported that potential differences in skin from individuals of varying age, pharmacokinetics with transdermal delivery may be altered. Other factors such as active ingredient physicochemical characteristics and formulation components, determine whether a specific drug will have pharmacokinetic differences across age groups [14]. This can be the basis for further studies of how transdermal glucosamine transport is affected by age, and shows the potential for age-specific glucosamine cream products with optimised transport efficiencies.

\subsection{Transdermal Glucosamine Absorption}

Successful detection of increased glucosamine concentrations in the synovial fluid of subjects show that glucosamine has permeated the skin layers and was most probably transported by specific transporters from blood. The study also shows that the knee is able to absorb glucosamine and that glucosamine can specifically enter a subject's synovial fluid. There is therefore potential for this to be applied to other joints. Although the mechanism for transdermal transport of glucosamine is not completely understood, it has been reported that GLUT2, one of the three glucose transporters (GLUT 1,2,4), has a high affinity for glucosamine and can actively transport exogenous glucosamine from extracellular tissue into cells [15] [16]. As such, GLUT2 may have a key role to play in the transport mechanism of glucosamine into cells. The synovial fluid is an ultra-filtrate of blood plasma that is concentrated by virtue of its filtration through 
the synovial membrane. The synovial matrix provides the permeable pathway through which exchange of molecules occurs, but also offers sufficient outflow resistance to retain large solutes of synovial fluid within the joint cavity [17].

It is scientifically established that water-soluble compounds like glucosamine cannot readily permeate through the lipophilic layers of the skin. The results of this study demonstrate the potential that if suitable vehicles are used to enable the transport of glucosamine, as in the case of TGC ${ }^{\circ}$ Plus Capsaicin, transdermal delivery might also be possible for other water-soluble compounds.

\subsection{Elimination of Outliers}

Only six outliers were identified (three in each group) and excluded from the main results. The HPLC analysis were repeated and it was confirmed that the results markedly deviated from other observations. Even if the outliers are included in the calculations, the average concentration of glucosamine of the Test group is still significantly higher $(145.84 \mathrm{ng} / \mathrm{ml}, 95 \%$ CI $80.26-211.41 ; \mathrm{n}=120)$ than the corresponding value for the Control group (35.92 ng/ml, 95\% CI 12.76 59.08, $\mathrm{n}=120$ ); at $\mathrm{P}=0.002$.

\subsection{Study Limitations}

The study design deliberately involved a large sample size to improve the confidence level of results obtained. The Control group acted as a proxy for a Time- 0 (T0) sample to establish a baseline of glucosamine level in the synovial fluid of subjects in the Test group. Ideally, a T0 sample should have been used as a control. As arthrocentesis is a relatively painful procedure, this study was designed, for ethical reasons, to avoid subjecting the patients to an additional extraction in order to obtain T0 data. Instead we have collected a significant number of samples from a control group that is already seeking arthrocentesis treatment as a proxy for T0 data, hence avoiding any additional pain.

As this study was not a pharmacokinetic study, the $\mathrm{T}_{\max }$ (time at which the highest concentration is observed) was unknown. As the synovial fluid samples for the Test group were collected one to three hours after the application of the glucosamine cream, the concentrations reported here are likely to be underestimated and could have been higher if the $\mathrm{T}_{\max }$ had been determined beforehand.

The synovial fluid volume of the normal human knee joint is $0.5-4 \mathrm{ml}$ but in pathological conditions it may exceed $100 \mathrm{ml}$ [18]. In our study, extracted synovial fluid volume for each participant varied from $2.5 \mathrm{ml}$ to $50 \mathrm{ml}$. Since the residual amount of synovial fluid after the arthrocentesis was unknown, we decided to measure and compare the glucosamine concentration rather than its absolute amount. A previous study showed that there was a large residual synovial fluid volume (mean 48\%), even after forced aspiration [19]. Methods do exist to measure the total synovial fluid volume [18] [20] but they were not appropriate for our clinical setting, because they involve additional injections. We have calculated another set of data (not shown) to include the volume as col- 
lected for each patient and this does not change the overall conclusions of the study. Assuming that the synovial fluid volume extracted is proportional to the total synovial fluid originally present, the absolute amount of glucosamine present in each sample was still significantly higher $(P=0.0093)$ for the Test group, compared to the Control group. Further research work will be necessary to measure the total volume of synovial fluid and get a more accurate profile of glucosamine level in synovial fluid.

\section{Conclusions and Recommendations for Further Studies}

This clinical study presented empirical evidence of transdermal delivery of glucosamine resulting in its bioavailability in the synovial fluid of test patients with OA of the knee. This study reinforces results from a prior unpublished pharmacokinetic study where glucosamine was detected in mice plasma 1 - 3 hours after topical glucosamine treatment. Whether the amount of glucosamine delivered is sufficient to be translated into therapeutic effect remains to be elucidated and could be the subject for future studies.

This study demonstrated that with a proper vehicle, a water-soluble molecule such as glucosamine can be delivered through human skin, and further studies may be conducted to investigate the potential transdermal delivery of other compounds using the same vehicle. The transdermal delivery route represents a highly attractive alternative to oral delivery, especially for demographic groups such as the elderly. The rate and mechanism of glucosamine absorption is not documented in this work, and further research is recommended on specific chemical properties of cream formulations that may control the glucosamine transdermal transport steps from a cream matrix, through skin layers.

\section{Acknowledgements}

The authors wish to thank Siti Norasikin for her assistance with numerous HPLC analyses, and Chua Yek Tann and Sheng Ping for their contributions to earlier analytical work.

\section{Conflicts of Interest}

Ting, C.M. has declared no conflicts of interest. Temasek Polytechnic, School of Applied Science was engaged by Lynk Biotechnologies Pte. Ltd. for data acquisition, analysis and interpretation.

\section{References}

[1] WHO (2019) Chronic Rheumatic Conditions: Osteoarthritis. http://www.who.int/chp/topics/rheumatic/en

[2] Hammad, Y.H., Hala, R.M. and Sobhy, M.M. (2015) Clinical and Biochemical Study of the Comparative Efficacy of Topical versus Oral Glucosamine/Chondroitin Sulfate on Osteoarthritis of the Knee. The Egyptian Rheumatologist, 37, 85-91. https://doi.org/10.1016/j.ejr.2014.06.007

[3] Leite, C.B.S., Coehlo, J.M., Muehlmann, L.A., de Azevedo, R.B. and Sousa, M.H. 
(2017) Skin Delivery of Glucosamine and Chondroitin Sulphates-A Perspective on the Conservative Treatment for Osteoarthritis of the Knee. Journal of Biosciences and Medicines, 5, 11-20. https://doi.org/10.4236/jbm.2017.54002

[4] Onigbinde, A.T., Talabi, A.E. and Shehu, R.A. (2011) Acute Effects of Combination of Glucosamine Sulphate Iontophoresis with Exercise on Fasting Plasma Glucose of Participants with Knee Osteoarthritis. Hong Kong Physiotherapy Journal, 29, 79-85. https://doi.org/10.1016/j.hkpj.2011.06.003

[5] Cohen, M., Wolfe, R., Mai, T. and Lewis, D. (2003) A Randomized, Double Blind, Controlled Trial of a Topical Cream Containing Glucosamine Sulfate, Chondroitin Sulfate, and Camphor for Osteoarthritis of the Knee. The Journal of Rheumatology, 30, 523-528.

[6] CDC (2018) Transparent Reporting of Evaluations with Non-Randomised Designs (TREND). https://www.cdc.gov/trendstatement/index.html

[7] YTL Community (2008) Suffering Worn-out, Osteo-Arthritic Knees? Cheah Ui-Hoon Finds out How to Avoid Knee Replacement Surgery.

http://www.ytlcommunity.com/commnews/shownews.asp?newsid=37518

[8] Ting, C.M. (2007) Advancing Visco Supplementation-A Handbook on Viscosupplementation for OA Knees. Armour Publishing Pte Ltd., Singapore.

[9] Persiani, S., Rotini, R., Trisolino, G., Rovati, L.C., Locatelli, M., Paganini, D., et al. (2007) Synovial and Plasma Glucosamine Concentrations in Osteoarthritic Patients Following Oral Crystalline Glucosamine Sulphate at Therapeutic Dose. Osteoarthritis Cartilage, 15, 764-772. https://doi.org/10.1016/j.joca.2007.01.019

[10] Pastorini, E., Rotini, R., Guardigli, M., Vecchiotti, S., Persiani, S., Trisolino, G., et al. (2009) Development and Validation of a HPLC-ES-MS/MS Method for the Determination of Glucosamine in Human Synovial Fluid. Journal of Pharmaceutical and Biomedical Analysis, 50, 1009-1014. https://doi.org/10.1016/j.jpba.2009.07.008

[11] Roy, S.D. and Flynn, G.L. (1990) Transdermal Delivery of Narcotic Analgesics: pH, Anatomical, and Subject Influences on Cutaneous Permeability of Fentanyl and Sufentanil. Pharmaceutical Research, 7, 842-847. https://doi.org/10.1023/A:1015912932416

[12] So, J., Ahn, J., Lee, T.H., Park, K.H., Paik, M.K., Jeong, M., et al. (2014) Comparison of International Guidelines of Dermal Absorption Tests Used in Pesticides Exposure Assessment for Operators. Toxicological Research, 30, 251-260. https://doi.org/10.5487/TR.2014.30.4.251

[13] Farage, M.A., Miller, K.W., Elsner, P. and Maibach, H.I. (2013) Characteristics of the Aging Skin. Advances in Wound Care, 2, 5-10. https://doi.org/10.1089/wound.2011.0356

[14] Konda, S., Meier-Davis, S.R., Cayme, B., Shudo, J. and Maibach, H.I. (2012) AgeRelated Percutaneous Penetration Part 2: Effect of Age on Dermatopharmacokinetics and Overview of Transdermal Products. Skin Therapy Letter, 17, 5-7.

[15] Anderson, J.W., Nicolosi, R.J. and Borzelleca, J.F. (2005) Glucosamine Effects in Humans: A Review of Effects on Glucose Metabolism, Side Effects, Safety Considerations and Efficacy. Food and Chemical Toxicology, 43, 187-201. https://doi.org/10.1016/j.fct.2004.11.006

[16] Uldry, M., Ibberson, M., Hosokawa, M. and Thorens, B. (2002) GLUT2 Is a High Affinity Glucosamine Transporter. FEBS Letters, 524, 199-203. https://doi.org/10.1016/S0014-5793(02)03058-2

[17] Tamer, T.M. (2013) Hyaluronan and Synovial Joint: Function, Distribution and Healing. Interdisciplinary Toxicology, 6, 111-125. 
https://doi.org/10.2478/intox-2013-0019

[18] Geborek, P., Saxne, T., Heinegard, D. and Wollheim, F.A. (1988) Measurement of Synovial Fluid Volume Using Albumin Dilution upon Intraarticular Saline Injection. The Journal of Rheumatology, 15, 91-94.

[19] Rekonen, A., Oka, M. and Kuikka, J. (1973) Measurement of Synovial Fluid Volume by a Radioisotope Method. Scandinavian Journal of Rheumatology, 2, 33-35. https://doi.org/10.1080/03009747309097148

[20] Kraus, V.B., Stabler, T.V., Kong, J., Varju, G. and McDaniel, G. (2007) Measurement of Synovial Fluid Volume Using Urea. Osteoarthritis Cartilage, 15, 1217-1220. https://doi.org/10.1016/j.joca.2007.03.017 